\title{
Analysis and Comparative Evaluation of Discrete Tangent Estimators
}

\author{
Jacques-Olivier Lachaud, Anne Vialard, and François de Vieilleville \\ LaBRI, Univ. Bordeaux 1, 351 cours de la Libération, \\ 33405 Talence Cedex, France \\ \{lachaud, vialard, devieill\}@labri.fr
}

\begin{abstract}
This paper presents a comparative evaluation of tangent estimators based on digital line recognition on digital curves. The comparison is carried out with a comprehensive set of criteria: accuracy on smooth or polygonal shapes, behaviour on convex/concave parts, computation time, isotropy, asymptotic convergence. We further propose a new estimator mixing the qualities of existing ones and outperforming them on most mentioned points.
\end{abstract}

\section{Introduction}

In this paper, we address the problem of tangent estimation along contours of digitized 2D objects. Tangent estimation has many applications in discrete geometry. For instance, the length of a digital contour is accurately estimated from tangents by integration 3, 9. Derivating the orientation of the tangent provides an estimation of the curvature 9, 11, 12. The previous geometric parameters are used in classical pattern recognition applications. They also define the internal energies of discrete deformable models [7]. When rendering 3D digitized objects, the normal vector field can be estimated from tangent directions along slice contours 8, 9].

When trying to estimate geometric properties of digitized objects, we face the issue that infinitely many shapes have the same digitization: there is no good approximation since there is no reference shape. Other hypotheses are thus required. The common assumption is that the original continuous object has some "natural" properties such as: compactness (not a fractal), bounded curvature, sometimes piecewise linear geometry (i.e. polygon). Therefore we restrict the class of shapes we are interested in. Discrete boundaries will come from the digitization of continuous shapes composed of polygonal parts and of smooth parts with bounded curvature.

Many tangent estimators are based on a fixed-size window of curve points around the point of interest [1, 9, 10, 12. However these methods cannot converge asymptotically to the value on the continuous shape because the computation scale is not adapted to the local shape geometry. This is why we take into account in this comparative analysis only estimators based on digital straight segment extraction which use an adaptative window size [6, 8, 11. 
In Section 2, we recall the existing definitions of discrete tangents and compare qualitatively their advantages and drawbacks. We then propose in Section 3 a new tangent estimator, called $\lambda-M S T$, that takes the best out of the existing ones. This estimator is based on the set of maximal digital straight segments going through the point of interest. We prove it has two interesting properties: it identifies convex and concave parts of the shape and behaves accordingly, its computational complexity is equivalent to the other existing estimators both locally and globally for the whole curve. Section 4 is devoted to an experimental comparative evaluation of the tangent estimators. We have checked the following points: tangent estimation on smooth and straight parts of the shape, sharp corner recognition, isotropy, mean and maximal asymptotical error with different shapes. The $\lambda$-MST appears to have the best behaviour in most practical cases.

\section{Estimating Tangent with Digital Straight Segments}

We restrict our study to the geometry of 4-connected digital curves. Indeed, a digital object is a set of pixels and its boundary when seen as a collection of pointels and linels is a 4-connected curve. Besides this work may easily be adapted to 8-connected curves since it relies on DSS recognition. We introduce some notations to get homogeneous definitions of existing tangent estimators based on digital straight lines. In the remaining of the paper, the digital curve is denoted by $C$. Its points $\left(C_{k}\right)$ are assumed to be indexed from 0 to $N-1$. A set of successive points of $C$ ordered increasingly from index $i$ to $j$ will be conveniently denoted by $C_{i, j}$.

\subsection{Standard Line, Digital Straight Segment, Maximal Segments}

Definition 1. The set of points $(x, y)$ of the digital plane verifying $\mu \leq a x-b y<$ $\mu+|a|+|b|$, with $a, b$ and $\mu$ integer numbers, is called the standard line with slope $a / b$ and shift $\mu$.

The standard lines are the 4-connected discrete lines. As we will see later, all discrete tangents are defined as particular connected subset of standard lines included in 4-connected digital curves.

Since the tangent is a local property of the curve, we can always assume that we look at a restricted part of $C$, where the indices are totally ordered (the curve can be re-indexed differently so that its indices are totally ordered on the subpart of interest). The following definition is thus valid.

Definition 2. We say that a set of successive points $C_{i, j}$ of the digital curve $C$ is a digital straight segment (DSS) iff there exists a standard line $(a, b, \mu)$ containing them. The predicate $C_{i, j}$ is a DSS is denoted by $S(i, j)$. When $S(i, j)$, we denote by $D(i, j)$ the characteristics associated with the digital straight segment [4]: the characteristics $(a, b, \mu)$ of the standard line containing all the points $C_{i, j}$, the end points $C_{i}$ and $C_{j}$, the principal upper and lower leaning points $U_{m}, U_{M}, L_{m}, L_{M}$. 
The first index $j, i \leq j$, such that $S(i, j)$ and $\neg S(i, j+1)$ is called the front of $i$. The map associating any $i$ to its front is denoted by $F$. Symmetrically, the first index $i$ such that $S(i, j)$ and $\neg S(i-1, j)$ is called the back of $j$ and the corresponding mapping is denoted by $B$.

The definition of maximal segments will be central for estimating tangents. They form the longest possible DSS in the curve. They are used for polygonizing the curve into the minimum number of segments [6].

Definition 3. Any set of points $C_{i, j}$ is called a maximal segment iff any of the following equivalent characterizations holds: (1) $S(i, j)$ and $\neg S(i, j+1)$ and $\neg S(i-1, j)$, (2) $B(j)=i$ and $F(i)=j$, (3) $\exists k, i=B(k)$ and $j=F(B(k))$, (4) $\exists k^{\prime}, i=B\left(F\left(k^{\prime}\right)\right)$ and $j=F\left(k^{\prime}\right)$.

\section{$2.2 \quad$ Discrete Tangents}

Based on local DSS recognition, several tangent estimators at a digital curve point have been proposed. Their quality is to adapt the computation window to the local shape of the curve. Exact tangent estimation for digitizations of straight lines can thus be achieved. They all try to make the right balance between longest and most centered DSS around the point of interest.

Definition 4. The following DSS may be defined around any point $C_{k}$ of the digital curve $C$. They correspond to the notion of discrete tangent (see Fig. 1).

- The DSS $C_{k-l, k+l}$ with $S(k-l, k+l)$ and $\neg S(k-l-1, k+l+1)$ is called the symmetric tangent $(\mathrm{ST})$ at $C_{k}$ [8].

- The maximal segment with biggest indices that includes the symmetric tangent at $C_{k}$ is called the Feschet-Tougne tangent (FTT) at $C_{k}$ [6].

- The extended tangent (ET) at $C_{k}$ includes the symmetric tangent $C_{k-l, k+l}$ but may be extended in the two following cases: (i) if $S(k-l, k+l+1) \wedge \neg S(k-$ $l-1, k+l)$ then it is extended forward as the maximal segment $C_{k-l, F(k-l)}$, (ii) if $S(k-l-1, k+l) \wedge \neg S(k-l, k+l+1)$ then it is extended backward as the maximal segment $C_{B(k+l), k+l}$.

- The forward half-tangent at $C_{k}$ is the DSS $C_{k, F(k)}$ and the backward halftangent at $C_{k}$ is the $D S S C_{B(k), k}$. The median half-tangent (HT) at $C_{k}$ is the arithmetical line median to the two half-tangents.

Any DSS defines an angle between its carrying standard line and the $x$-axis (in $[0 ; 2 \pi$ [ since a DSS is oriented). This angle will be called later on the direction of the DSS and denoted by the symbol $\theta$.

The preceding discrete tangent definitions, except for the FTT, are independent of the orientation chosen for the curve (Fig. 1 $d-e$ ). ET can be seen as an unambiguous version of FTT. Both FTT and ET are local longest DSS, to the expense of a loss of localization around the point. FTT and ET tend to polygonalize the digital curve even for underlying smooth shapes.

On the other hand, ST and HT have a very good localization around the point (perfectly centered for ST). However they both may have a bad behavior on even very regular shapes (e.g. at the points where a circle with integer 

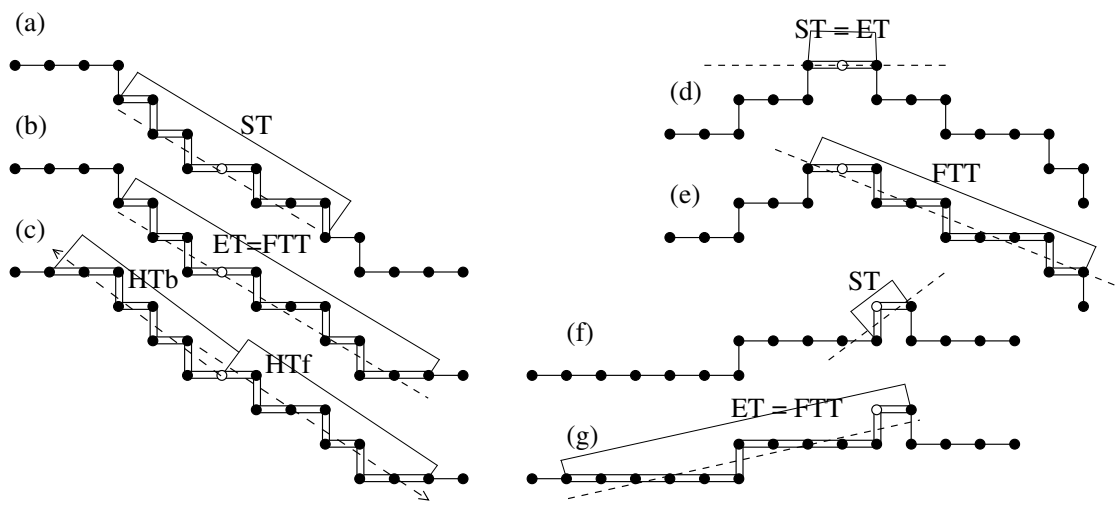

Fig. 1. Illustration of discrete tangents. (a) ST. (b) ET = FTT (here). (c) forward and backward HT. Subfigures d-g show specific problem raised by FTT and ST and solved by ET: (de) balanced tangent for ET and ST versus arbitrarily unbalanced tangent for FTT, (fg) false concavity detected by ST versus correct straight line for ET and FTT

radii touches the axes). They may also not locate accurately convex or concave parts of the curve (Fig. 1f-g). Note HT is also used for computing the curvature [2].

It is thus not clear which definition of discrete tangent is the best suited to a given application. A comparative evaluation of all tangent definitions is thus necessary to anticipate their behavior for given shapes and applications. This evaluation is made in Section 4. Before that, we construct a new tangent estimator which aims at mixing the qualities of the other ones: related to maximal segments as FTT and ET; computation window identical to HT; significant position of the point wrt the DSS surrounding it as ST; unambiguous definition.

\section{Tangent Estimation Based on Maximal Segments}

We define a new tangent estimator that depends on the set of maximal segments that goes through a point of the digital curve. This set is called the pencil of maximal segments around the point of interest. As noted by Feschet and Tougne [6], several successive points may have the same pencil. Therefore the tangent estimator takes also into account the position of the point within the pencil. More specifically, the point has a given eccentricity wrt each maximal segments. The tangent direction is estimated by a combination of the direction of each maximal segment weighted by the eccentricity. In the following subsections, we formalize the new tangent estimator, we then show it preserves convexity/concavity with minor restrictions and we explicit lastly how to compute it in optimal time. 


\subsection{Eccentricity, Maximal Segment Tangent Estimator}

We index all the maximal segments of the curve by increasing indices: $M^{i}=$ $C_{m_{i}, n_{i}}$ with $F\left(m_{i}\right)=n_{i}$ and $B\left(n_{i}\right)=m_{i}$. From characterizations (3) and (4) of the definition of maximal segment (Definition 3), any DSS $C_{i, j}$ and hence any point belongs to at least two maximal segments $C_{B(j), F(B(j))}$ and $C_{B(F(i)), F(i)}$. Therefore, the pencil of maximal segments $\mathcal{P}(k)=\left\{M^{i}, k \in M^{i}\right\}$ around any point $C_{k}$ is never empty. We denote by $\theta_{i}$ the direction of the DSS $M^{i}$. In the remaining of the paper, $\lambda$ is a mapping from $[0,1]$ to $\mathbb{R}^{+}$with $\lambda(0)=\lambda(1)=0$ and $\lambda>0$ elsewhere.

The eccentricity of $C_{k}$ wrt a maximal segment $M^{i}$ is defined as

$$
e_{i}(k)=\left\{\begin{array}{ll}
\frac{\left\|C_{k}-C_{m_{i}}\right\|_{1}}{L_{i}}=\frac{k-m_{i}}{L_{i}} & \text { if } i \in \mathcal{P}(k) \\
0 & \text { otherwise }
\end{array}, \text { with } L_{i}=\left\|C_{n_{i}}-C_{m_{i}}\right\|_{1} .\right.
$$

Definition 5. The $\lambda$-maximal segment tangent direction at point $C_{k}(\lambda$-MST) is defined as

$$
\hat{\theta}(k)=\frac{\sum_{i \in \mathcal{P}(k)} \lambda\left(e_{i}(k)\right) \theta_{i}}{\sum_{i \in \mathcal{P}(k)} \lambda\left(e_{i}(k)\right)}=\frac{\sum_{i} \lambda\left(e_{i}(k)\right) \theta_{i}}{\sum_{i} \lambda\left(e_{i}(k)\right)} .
$$

Considering the properties of the eccentricity and the non-emptyness of pencils, this value is always defined and may be computed locally.

The preceding notion is extended to any real value $k$ in $[0, N[$. It is enough to consider $k$ as the curvilinear parameterization of the 4-connected contour. Any non-integer value of $k$ corresponds to a real point on the straight line linking $C_{\lfloor k\rfloor}$ and $C_{\lceil k\rceil}$. When $\lambda$ is continuous, the angle $\hat{\theta}(k)$ is continuous too and a length estimator may be derived from it, giving $\hat{l}(k)=\frac{h}{|\cos (\hat{\theta}(k))|+|\sin (\hat{\theta}(k))|}$. The length of the curve can be estimated by simple integration of $\hat{l}(k)$. Coeurjolly and Klette have reported that this method of length evaluation gives very good results 3 .

\subsection{Local Convexity or Concavity; Characterization of $\lambda$}

Feschet proposes to use maximal segments for decomposing the curve into convex and concave parts 5 . The following definition shares the same idea.

Definition 6. The digital curve $C$ is oriented counterclockwise wrt the discrete object it bounds. $C$ is locally convex (resp. concave) at point $C_{k}$ iff the angles $\left(\theta_{i}\right)$ of the sorted segments of $\mathcal{P}(k)$ is an nondecreasing sequence (resp. nonincreasing sequence). (Angles are brought back in $]-\pi, \pi[$ relatively to the first one.)

We say that a tangent estimator to a digital curve satisfies the convexity/concavity property iff the estimated tangent direction is nondecreasing (resp. nonincreasing) on every connected subset where the curve is locally convex (resp. concave). This property holds for ET and FTT but does not hold for ST and HT (e.g. see Fig. 11). For $\lambda$-MST, it depends on the function $\lambda$ as indicated below. 
Theorem 1. If $\lambda$ is differentiable on $] 0,1[$, then the $\lambda$-MST estimator satisfies the convexity/concavity property iff $\frac{d}{d t}\left(t \frac{\lambda^{\prime}}{\lambda}(t)\right) \leq 0$ and $\frac{d}{d t}\left((1-t) \frac{\lambda^{\prime}}{\lambda}(t)\right) \leq 0$ hold on this interval.

The proof is given in appendix. It is easy to check that functions with a bell shape satisfy this constraint (e.g. functions based on binomials). This is for instance the case for the $C^{2}$ function $64\left(-x^{6}+3 x^{5}-3 x^{4}+x^{3}\right)$ or for the $C^{\infty}$ function $\exp \left(4-\frac{1}{x}-\frac{1}{1-x}\right)$ extended by zeroes. One may also find functions not differentiable everywhere which satisfies the convexity/concavity. Among them, we can quote the triangle function with a peak at $\frac{1}{2}$.

\subsection{Complexity Issues}

Another interesting criterion for choosing a tangent estimation is its computational cost. Feschet and Tougne [6] showed an algorithm that computes the FTT to all points of a curve in a time linear with the number of points. We show here that all maximal segments of a curve can be computed with the same complexity. The $\lambda$-MST to all points of a curve is thus quickly computed.

Given a maximal segment $M^{k}=C_{m_{k}, n_{k}}$, its next maximal segment can be defined as $C_{B\left(n_{k}+1\right), F\left(B\left(n_{k}+1\right)\right)}$. It is the maximal segment containing the point $n_{k}+1$ and obtained from $M^{k}$ with a minimal number of operations (adding and removing a point). The following algorithm computes it:

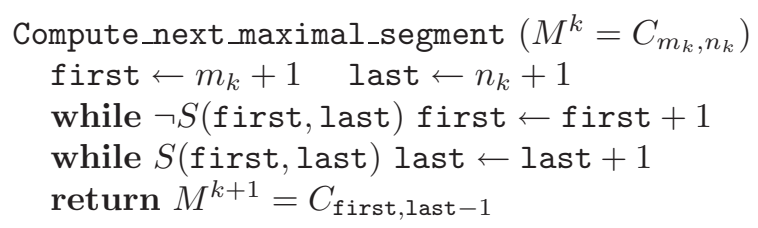

Its principle is to remove points at the backward extremity of $M^{k}$ until it becomes possible to extend the resulting segment at the other end. Of course, the characteristics of the intermediate DSS must be updated at each removal or addition of a point. The time complexity of the preceding function depends on the complexity of the updates, which are proved to be $O(1)$ by:

Theorem 2. Assume $S(i, j)$, and assume the characteristics $D(i, j)$ of the corresponding DSS are known. Then,

1. (Addition of point $C_{i}$ or $\left.C_{j}\right)$ deciding $S(i, j+1)$ or $S(i-1, j)$ are $O(1)$ operations and, when appropriate, computing $D(i, j+1)$ or $D(i-1, j)$ are $O(1)$ operations too (proved by Debled-Renesson and Réveillès [4]);

2. (Removal of point $C_{i}$ or $\left.C_{j}\right)$ computing $D(i+1, j)$ or $D(i, j-1)$ are $O(1)$ operations (see below).

An immediate corollary is that all the maximal segments of a given closed digital curve are computed with a linear complexity (each point of the curve is added once to a segment and removed once). Remark that the complexity of 

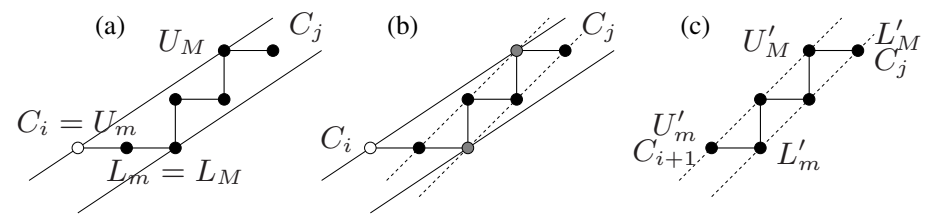

Fig. 2. Removal of a point from a DSS. (a) DSS $C_{i, j}$. The point $C_{i}$ is an upper leaning point and its removal will increase the segment slope. (b) Rotation of the leaning lines around the pivot points (in gray) during the addition/removal of $C_{i}$. (c) DSS $C_{i+1, j}$. Its slope and the leaning points $U_{m}^{\prime}$ and $L_{M}^{\prime}$ have to be recomputed

computing the pencil $\mathcal{P}(k)$ around $C_{k}$ depends on the local shape of the curve $(O(F(k)-B(k)))$. We now explain briefly how to update a DSS in constant time when removing a point.

Let $C_{i, j}$ be a DSS of characteristics $D(i, j)=\left(a, b, \mu, U_{m}, U_{M}, L_{m}, L_{M}\right)$. Let us recall that $U_{m}, U_{M}, L_{m}$ and $L_{M}$ are leaning points that belong to the DSS, e.g. their remainder equal $\mu$ or $\mu+|a|+|b|-1$. $U_{m}$ and $U_{M}$ (resp. $L_{m}$ and $L_{M}$ ) are upper (resp. lower) leaning points, leftmost and rightmost. Without any loss in generality this digital segment belongs to the first quadrant. In the following, we denote by $\left(a^{\prime}, b^{\prime}, \mu^{\prime}, U_{m}^{\prime}, U_{M}^{\prime}, L_{m}^{\prime}, L_{M}^{\prime}\right)$ the characteristics $D(i+1, j)$ of the DSS $C_{i+1, j}$, which we wish to compute. Our algorithm is based on the observation that if the addition of the point $C_{i}$ to $C_{i+1, j}$ has changed the characteristics $D(i+1, j)$, its removal from $C_{i, j}$ should do an inverse modification to $D(i, j)$. After the examination of the incremental algorithm in 4 which explains how to update the characteristics when a point is added, this situation happens when $C_{i}$ is an upper or lower leaning point of the DSS $C_{i, j}$. Fig. 2 illustrates the case where $C_{i}$ is an upper leaning point.

We detail here the update when $C_{i}=U_{m}$ and $\overrightarrow{C_{i} U_{M}}=(b, a)$ and $L_{m}=$ $L_{M}$. Clearly, the addition of $C_{i}$ to $C_{i+1, j}$ has decreased the slope of the DSS. Geometrically, it corresponds to a rotation of the upper leaning line around $U_{M}^{\prime}$ and of the lower leaning line around $L_{m}^{\prime}$. The two leaning points $U_{M}$ and $L_{m}$ are thus left unchanged by the removal of $C_{i}$. We can also easily state that the point $P=\left(x_{C_{i}}+1, y_{C_{i}}-1\right)$ would have extended $C_{i+1, j}$ without modifying its characteristics $D(i+1, j)$. The values $\left(a^{\prime}, b^{\prime}, \mu^{\prime}\right)$ are deduced from $P$. The

Table 1. Updates of $D(i, j)$ when removing point $C_{i}$

\begin{tabular}{|c|c|c|}
\hline & $C_{i}=U_{m} \wedge \overrightarrow{C_{i} U_{M}}=(b, a) \wedge L_{m}=L_{M}$ & $C_{i}=L_{m} \wedge \overrightarrow{C_{i} L_{M}}=(b, a) \wedge U_{m}=U_{M}$ \\
\hline \hline$a^{\prime}$ & $y_{L_{m}}-\left(y_{C_{i}}-1\right)$ & $y_{U_{m}}-\left(y_{C_{i}}+1\right)$ \\
\hline$b^{\prime}$ & $x_{L_{m}}-\left(x_{C_{i}}+1\right)$ & $x_{U_{m}}-\left(x_{C_{i}}-1\right)$ \\
\hline$\mu^{\prime}$ & $a^{\prime} x_{U_{M}}-b^{\prime} y_{U_{M}}$ & $a^{\prime} x_{U_{m}}-b^{\prime} y_{U_{m}}$ \\
\hline$U_{m}^{\prime}$ & $U_{M}-\left(x_{U_{M}}-x_{C_{i}}-1\right) / b^{\prime}\left(b^{\prime}, a^{\prime}\right)$ & $U_{m}$ \\
\hline$U_{M}^{\prime}$ & $U_{M}$ & $U_{m}+\left(\left(y_{C_{j}}-y_{C_{i}}-1\right) / a^{\prime}-1\right)\left(b^{\prime}, a^{\prime}\right)$ \\
\hline$L_{m}^{\prime}$ & $L_{m}$ & $L_{M}-\left(y_{L_{M}}-y_{C_{i}}-1\right) / a^{\prime}\left(b^{\prime}, a^{\prime}\right)$ \\
\hline$L_{M}^{\prime}$ & $L_{m}+\left(\left(x_{C_{j}}-x_{C_{i}}-1\right) / b^{\prime}-1\right)\left(b^{\prime}, a^{\prime}\right)$ & $L_{M}$ \\
\hline
\end{tabular}


updating of $U_{m}$ and $L_{M}$ is a little more tricky and exploits the property that the vector linking two successive upper (or lower) leaning points is $\left(b^{\prime}, a^{\prime}\right)$. The computation of the characteristics $D(i+1, j)$ are sumed up in the first column of Table 1. Its second column corresponds to the case where $C_{i}$ is a lower leaning point and its removal decreases the slope.

\section{Experimental Evaluation}

In this section, we perform a quantitative evaluation of tangent estimators based on DSS recognition. The behaviour of the $\lambda$-MST estimator rely on the $\lambda$ function which monitor the estimation of the underlying curve. For example recontruct$\operatorname{ing} C^{\infty}$ functions requires $C^{\infty} \lambda$ functions. We choose to minimize the curvature of the underlying curve by taking the symmetric triangle function with a peak at $\frac{1}{2}$ as $\lambda$ function. This function estimates the continuous underlying curve as a circular arc when the pencil of maximal segments is reduced to two maximal segments. Moreover it gives very good practical results. In the computer implementation, all tangent directions are estimated wrt linels, not points (i.e.
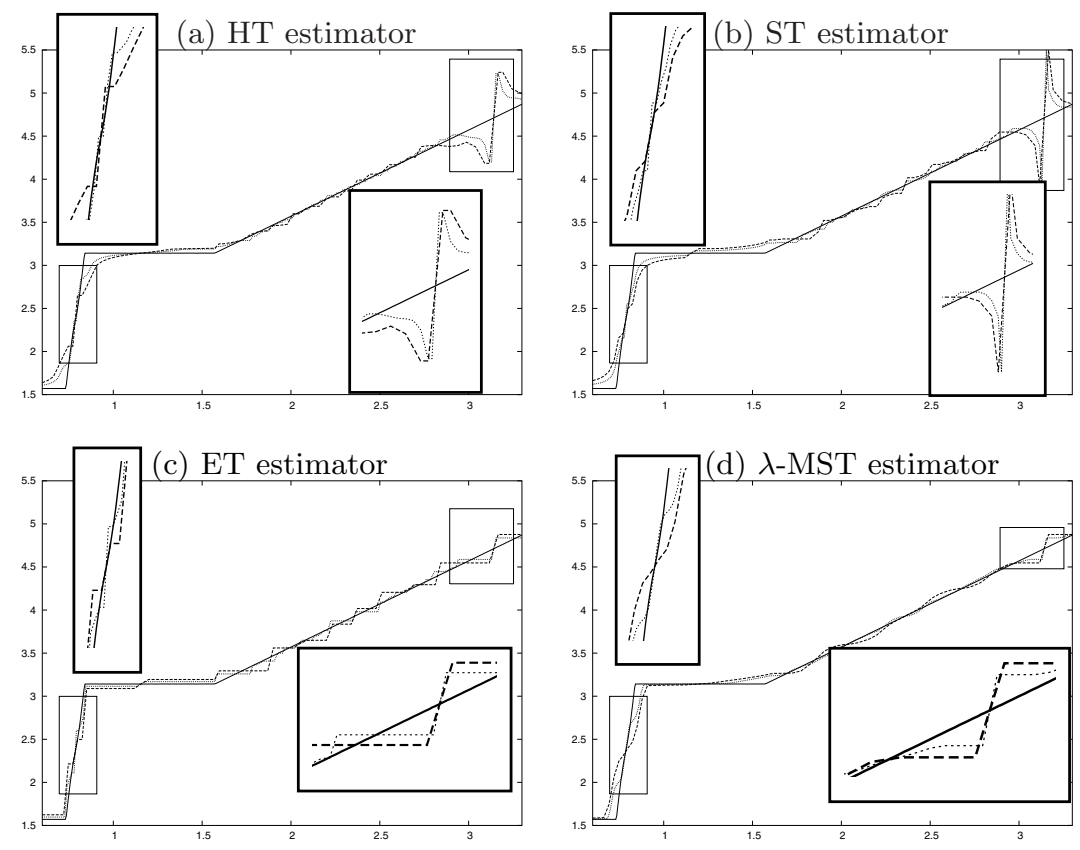

Fig. 3. Plots of the estimated tangent direction as a function of the polar angle. The shape is a circle of radius 10 with a sharp corner in the first quadrant. Solid lines correspond to expected values, dashed lines to estimations with a grid step of 0.5 , dotted lines to estimations with a finer grid step of 0.25 . 

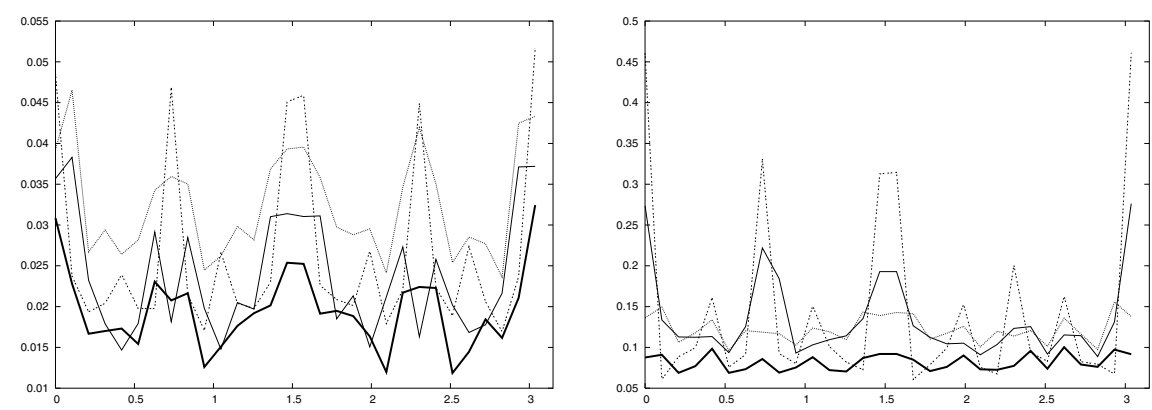

Fig. 4. Isotropy of tangent estimators measured with absolute error $|\hat{\theta}(t)-\theta(t)|$ (thick solid line: $\lambda$-MST, thin solid line: HT, dashed line: ST, dotted line: ET). Left: mean of absolute error. Right: Maximum of absolute error. For each estimator, 100 experiments are run on a circle of radius 50 with a center arbitrarily shifted in its pixel. The absolute error is drawn as a function of the polar angle and gathered by sectors of $\frac{5}{180} \pi$

geometric quantities are computed at curvilinear abscissa $k+\frac{1}{2}$ and all DSS includes $k$ and $k+1$ ).

We first compare the behavior of tangent estimators on smooth and flat parts and on corners. The shape is a circle in three quadrants and a right angle in the fourth (see "rsquare" in Table 2). Fig. 3 displays (a subset of) the estimations of the tangent direction. Estimators that satisfies the convexity/concavity property, i.e. ET and $\lambda$-MST, create a non-decreasing sequence of directions. ST and HT

Table 2. Asymptotic convergence of mean and maximum absolute error for tangent estimators. Best estimator is shaded. The grid step is denoted by $h$. The maximum and minimum curvatures of the shapes are: "circle" $\left(\kappa_{M}=\kappa_{m}=1\right)$, "flower" $\left(\kappa_{M} \approx\right.$ $\left.5.8, \kappa_{m} \approx-26.1\right)$, "rsquare" $\left(\kappa_{M}=100, \kappa_{m}=0\right)$

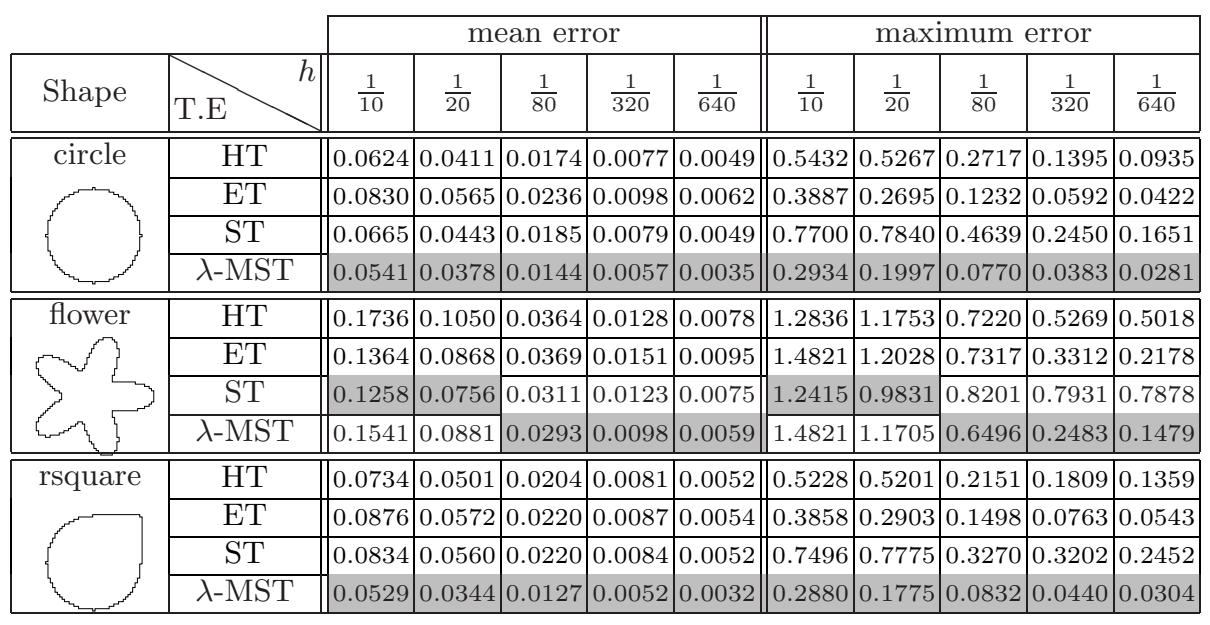


clearly fail, especially at points where the digital contour meets a quadrant change. Most estimators behave correctly at corners. $\lambda$-MST slightly smoothes the corner at low resolution. The tendency to polygonalize the curve of ET (and thus FTT) appears clearly on Fig. 3.

We then evaluate the anisotropy of the estimators with the experiment described in Fig. 4. The $\lambda$-MST is more isotropic than the others, with a steady and low mean and maximal error.

We finally examine the asymptotic behavior of the absolute error for different shapes on Table 2. Both $\lambda$-MST and ET have an asymptotic convergence in mean and in maximum. It is however unclear whether the maximum error of ST and HT converges toward 0 or not for arbitrary shapes. Although the $\lambda$-MST is not always the best in mean at coarse resolution, it has the fastest asymptotic convergence in mean and in maximum whatever the shape is.

\section{Conclusions}

In this paper, we have compared several tangent estimators based on DSS recognition. After a first qualitative analysis, we have proposed a new estimator which takes the best out of the existing ones. We have checked that it satisfies the convexity/concavity property and we have shown how to compute it efficiently. After experimental evaluation, the $\lambda$-MST appears to be the most robust tangent estimator and very often the most accurate. The results are summed up in Table 3. Future work will focus on curvature estimators based on maximal segments and their properties.

Table 3. Comparison of discrete tangent estimators. The $\lambda$-MST estimator has an average behaviour on corners and seems to be the best elsewhere

\begin{tabular}{|c||c|c|c|c|c|c|c|}
\hline $\begin{array}{c}\text { tangent } \\
\text { estimator }\end{array}$ & $\begin{array}{c}\text { straight } \\
\text { parts }\end{array}$ & $\begin{array}{c}\text { smooth } \\
\text { parts }\end{array}$ & corners & $\begin{array}{c}\text { convexity } \\
\text { /concavity }\end{array}$ & isotropy & $\begin{array}{c}\text { mean } \\
\text { error }\end{array}$ & $\begin{array}{c}\text { maximal } \\
\text { error }\end{array}$ \\
\hline \hline$\lambda$-MST & + & + & $=$ & Yes $^{*}$ & + & ++ & ++ \\
\hline HT & $=$ & $+/-$ & + & No & - & + & - \\
\hline ET & + & $=$ & + & Yes & $=$ & + & + \\
\hline ST & $=$ & $+/-$ & $=$ & No & - & + & - \\
\hline
\end{tabular}

$(*)$ For $\lambda$ functions satisfying conditions of Theorem [1]

\section{References}

1. I.M. Anderson and J.C. Bezdek. Curvature and tangential deflection of discrete arcs: a theory based on the commutator of scatter matrix pairs and its application to vertex detection in planar shape data. IEEE Transactions on Pattern Analysis and Machine Intelligence, 6:27-40, 1984.

2. D. Coeurjolly. Algorithmique et géométrie discrète pour la caractérisation des courbes et des surfaces. PhD thesis, Université Lumière Lyon 2, France, 2002. 
3. D. Coeurjolly and R. Klette. A comparative evaluation of length estimators of digital curves. IEEE Transactions on Pattern Analysis and Machine Intelligence, 26(2):252-258, 2004.

4. I. Debled-Renesson and J.-P. Réveillès. A linear algorithm for segmentation of discrete curves. International Journal of Pattern Recognition and Artificial Intelligence, 9:635-662, 1995.

5. F. Feschet. Canonical representations of discrete curves. Pattern Analysis and Application. Submitted.

6. F. Feschet and L. Tougne. Optimal time computation of the tangent of a discrete curve: Application to the curvature. In Proc. DGCI'99, volume 1568 of LNCS, pages 31-40. Springer, 1999.

7. J.-O. Lachaud and A. Vialard. Discrete deformable boundaries for the segmentation of multidimensional images. In Proc. 4th Int. Workshop on Visual Form, volume 2059 of $L N C S$, pages 542-551. Springer, 2001.

8. J.-O. Lachaud and A. Vialard. Geometric measures on arbitrary dimensional digital surfaces. In Proc. DGCI'03, volume 2886 of $L N C S$, pages 434-443. Springer, 2003.

9. A. Lenoir, R. Malgouyres, and M. Revenu. Fast computation of the normal vector field of the surface of a 3D discrete object. In Proc. DGCI'96, volume 1176 of LNCS, pages 101-112. Springer, 1996.

10. J. Matas, Z. Shao, and J. Kittler. Estimation of curvature and tangent direction by median filtered differencing. In Proc. of 8th International Conference on Image Analysis and Processing, pages 83-88, 1995.

11. A. Vialard. Geometrical parameters extraction from discrete paths. In Proc. DGCI'96, volume 1176 of LNCS, pages 24-35. Springer, 1996.

12. M. Worring and A. W. M. Smeulders. Digital curvature estimation. CVGIP: Image Understanding, 58:366-382, 1993.

\section{A Proof of Theorem 1}

We show here a necessary and sufficient condition for the $\lambda$ function to define a $\lambda$-MST tangent estimator satisfying the convexity/concavity property.

Theorem 1. If $\lambda$ is differentiable on ]0,1[, then the $\lambda$-MST estimator satisfies the convexity/concavity property iff $\frac{d}{d t}\left(t \frac{\lambda^{\prime}}{\lambda}(t)\right) \leq 0$ and $\frac{d}{d t}\left((1-t) \frac{\lambda^{\prime}}{\lambda}(t)\right) \leq 0$ hold on this interval.

These two conditions once put together entail $\lambda$ is necessarily log-concave (i.e. $\ln \lambda$ is a concave function or $\left.\frac{d^{2}}{d t^{2}}(\ln \lambda(t)) \leq 0\right)$. Furthermore, it is enough to check $\frac{d}{d t}\left(t \frac{\lambda^{\prime}}{\lambda}(t)\right) \leq 0$ for functions symmetric around $\frac{1}{2}$.

Proof. We first rewrite $\hat{\theta}^{\prime}(k)$ as

$$
\frac{\sum_{i<j}\left(\theta_{i}-\theta_{j}\right)\left(\frac{\lambda\left(e_{j}(k)\right) \lambda^{\prime}\left(e_{i}(k)\right)}{L_{i}}-\frac{\lambda\left(e_{i}(k)\right) \lambda^{\prime}\left(e_{j}(k)\right)}{L_{j}}\right)}{\left(\sum_{j} \lambda\left(e_{j}(k)\right)\right)^{2}} .
$$

We assume for instance that the angles $\left(\theta_{i}\right)$ of the segment in the pencil around $k$ are nondecreasing. We must thus prove $\hat{\theta}^{\prime}(k)$ is nonnegative, whatever is the 
curve under examination. Since some curves have points with exactly two maximal segments going through, Eq. (3) may be reduced to one pair. It is thus necessary to show that each term of this sum is nonnegative. It is also a sufficient condition. Otherwise said, we have to prove for any $i<j$,

$$
\forall k, m_{j}<k<n_{i}, \frac{\lambda\left(e_{j}(k)\right) \lambda^{\prime}\left(e_{i}(k)\right)}{L_{i}}-\frac{\lambda\left(e_{i}(k)\right) \lambda^{\prime}\left(e_{j}(k)\right)}{L_{j}} \leq 0 .
$$

Let $R_{i j}=n_{i}-m_{j}$ be the size of the common part of both segments. Setting $t=\frac{k-m_{j}}{R_{i j}}$, we define two analogs of the eccentricities $e_{i}(k)$ and $e_{j}(k)$ as $\epsilon_{i}(t)=$ $e_{i}(k)=1-\frac{R_{i j}}{L_{i}}(1-t)$ and $\epsilon_{j}(t)=e_{j}(k)=\frac{R_{i j}}{L_{j}} t$. Eq. (44) is then equivalent to

$$
\begin{array}{r}
\forall t \in] 0,1\left[, \quad \lambda\left(\epsilon_{j}(t)\right) \frac{\lambda^{\prime}\left(\epsilon_{i}(t)\right)}{L_{i}} \leq \lambda\left(\epsilon_{i}(t)\right) \frac{\lambda^{\prime}\left(\epsilon_{j}(t)\right)}{L_{j}}\right. \\
\Leftrightarrow \frac{R_{i j}}{L_{i}} \frac{\lambda^{\prime}}{\lambda}\left(\epsilon_{i}(t)\right) \leq \frac{R_{i j}}{L_{j}} \frac{\lambda^{\prime}}{\lambda}\left(\epsilon_{j}(t)\right) \Leftrightarrow \frac{d}{d t}\left(\ln \lambda\left(\epsilon_{i}(t)\right)\right) \leq \frac{d}{d t}\left(\ln \lambda\left(\epsilon_{j}(t)\right)\right)
\end{array}
$$

It is easy to see that $\epsilon_{i}(t)>t>\epsilon_{j}(t)$ which gives the idea to break Eq. (6) in two parts as follows, for all $t \in] 0,1[$ :

$$
\frac{d}{d t}\left(\ln \lambda\left(\epsilon_{i}(t)\right)\right) \leq \frac{d}{d t}(\ln \lambda(t)) \text { and } \frac{d}{d t}(\ln \lambda(t)) \leq \frac{d}{d t}\left(\ln \lambda\left(\epsilon_{j}(t)\right)\right)
$$

Eq. (7) clearly implies Eq. (6), but the converse is also true by letting $L_{i}$ or $L_{j}$ tend toward $R_{i j}$.

We focus on the right part of Eq. (77). Letting $\delta=\frac{R_{i j}}{L_{j}}$ and $f=\ln \lambda$, we get

$$
\forall \delta, 0<\delta<1, \frac{d}{d t}(f(t)) \leq \frac{d}{d t}(f(\delta t)) \text {, otherwise said } f^{\prime}(t) \leq \delta f^{\prime}(\delta t) .
$$

We now show that Eq. (8) is equivalent to

$$
\frac{d}{d t}\left(t f^{\prime}(t)\right) \leq 0
$$

Indeed, integrating both terms of the last inequality between $\delta t$ and $t$ shows sufficiency. It is also necessary since Eq. (8) can be rewritten with $h=(1-\delta) t$ as:

$$
\begin{array}{r}
f^{\prime}(t) \leq\left(1-\frac{h}{t}\right) f^{\prime}(t-h) \\
\frac{f^{\prime}(t)-f^{\prime}(t-h)}{h}+\frac{f^{\prime}(t-h)}{t} \leq 0
\end{array}
$$

Getting the limit when $h$ tends toward 0 and multiplying both sides by $t$ give $t f^{\prime \prime}(t)+f^{\prime}(t) \leq 0$, which is exactly Eq. (9). Same reasoning applied to left part of Eq. (7) bring

$$
\frac{d}{d t}\left((1-t) f^{\prime}(t)\right) \leq 0,
$$

which concludes the proof. 